\title{
The prevalence of left atrial enlargement in Polish patients with atrial fibrillation - a single center study
}

\author{
Anna Szymańska', Anna E. Płatek², Joanna Syska-Sumińska', Anna Gorajek', Mirosław Dłużniewski' \\ 'Department of Heart Diseases, Medial Centre of Postgraduate Education, Warsaw, Poland \\ ${ }^{2}$ Department of General and Experimental Pathology with Centre for Preclinical Research and Technology (CEPT), Medical University of Warsaw, \\ Warsaw, Poland
}

\begin{abstract}
Background. Atrial fibrillation (AF) remains one of the major causes of cardiovascular morbidity worldwide. Left atrial enlargement (LAE) is a common risk factor of AF. Left atrial enlargement is also connected with a higher prevalence of heart failure in AF patients. The aim of this study was to assess the prevalence of LAE in Polish patients with $\mathrm{AF}$.

Material and methods. Transthoracic echocardiography was performed in consecutive AF patients hospitalized in the Department of Heart Diseases. We assessed LAE using a two-dimensional method. Left atrial (LA) size was classified into the 4 categories: normal (LAE values $<39 \mathrm{~mm}$ in women and $<41 \mathrm{~mm}$ in men), mildly enlarged (39-42 $\mathrm{mm}$ in women and $41-46 \mathrm{~mm}$ in men), moderately enlarged (43-46 $\mathrm{mm}$ in women and $47-51 \mathrm{~mm}$ in men), and severely enlarged ( $\geq 47 \mathrm{~mm}$ in women and $\geq 52 \mathrm{~mm}$ in men).

Results. We analyzed 113 individuals with AF (mean age $77.2 \pm 9.8$ years; $37.2 \%$ men). Of these, $71(62.8 \%)$ patients had LAE (age $77.6 \pm 9.9$ years; $36.6 \% \mathrm{men}$ ). LA was mildly enlarged $(39-42 \mathrm{~mm}$ in women and $41-46 \mathrm{~mm}$ in men) in $20(28.2 \%)$ patients, moderately enlarged ( $43-46 \mathrm{~mm}$ in women and $47-51 \mathrm{~mm}$ in men) was observed in $30(42.3 \%)$, and severely enlarged ( $\geq 47 \mathrm{~mm}$ in women and $\geq 52 \mathrm{~mm}$ in men) in $21(29.6 \%)$ patients. The incidence of heart failure was significantly higher in AF patients with LAE 39 (54.9\%) compared to the patients without LAE - $12(28.6 \%) \mathrm{p}=0.01]$.

Conclusions. In patients with AF, LAE was highly prevalent. Patients with AF and LAE have more often HF compared to AF patients without LAE.

Key words: left atrial enlargement; atrial fibrillation; echocardiography
\end{abstract}

Arterial Hypertens. 2019, vol. 23, no. 4, pages: 271-274 DOI: $10.5603 /$ AH.a2019.0020

Address for correspondence: Anna Szymańska, MD, PhD

Department of Heart Diseases, Medial Centre of Postgraduate Education, Poznanska 22 Street, Warsaw 00-685, Poland;

tel: +48 22 5251263; e-mail: anna.szymanska@cmkp.edu.pl

V M Copyright (C) 2019 Via Medica, ISSN 2449-6170 


\section{Introduction}

Atrial fibrillation (AF), the most common supraventricular arrhythmia, is found in approximately 3\% adults and its prevalence is still rising. Atrial fibrillation remains one of the major causes of stroke, heart failure (HF), sudden death, and cardiovascular morbidity worldwide [1]. It occurs more often in patients with a left atrial enlargement (LEA) [2]. Left atrial (LA) size is a predictor of cardiovascular outcomes in the general population and HF patients [3]. As previously shown, LAE is also connected with higher thromboembolic risk in AF patients [4].

The aim of this study was to assess the prevalence of LAE in patients with nonvalvular AF.

\section{Material and methods}

The study was designed and conducted with the accordance of the Declaration of Helsinki and it was approved by the Regional Ethics Committee. We prospectively analyzed data on continuous hospitalizations for AF in the Department of Heart Diseases, Medial Centre of Postgraduate Education, Warsaw. Inclusion criteria were age $\geq 18$ years, primary diagnosis of AF, transthoracic echocardiographic evaluation underwent during current hospitalization. We excluded patients with valvular AF, fatal condition with an estimated life expectancy of $<6$ months, or those who did not give informed consent for the participation in the study. Medical history was taken in all patients on admission by a qualified physician. Data included information on thromboembolic and cardiovascular risk factors. The diagnosis of AF was made based on the rhythm documentation using a standard electrocardiogram (ECG) showing the typical pattern of AF, according to the current guidelines [2].

In all, transthoracic echocardiograms were performed in the Department's Echocardiography Laboratory using Philips Affiniti 70C Ultrasound Machines (Philips Healthcare, Andover, USA) by qualified echocardiographers. To avoid bias, measurements were taken three times, then averaged. LA diameter was measured using two-dimensional (2D) echocardiography, from the posterior aortic wall to the posterior left atrial wall, in the parasternal long-axis view at the end-ventricular systole (i.e., just before the mitral valve opening). Left atrial enlargement was classified into four categories: normal, mildly enlarged, moderately enlarged, and severely enlarged by the European Society of Cardiology guidelines [5]. Left atrial size was considered normal when LA was $<39 \mathrm{~mm}$ in women and $<41 \mathrm{~mm}$ in men, otherwise it was considered enlarged. The mild enlargement was defined as LAE values $39-42 \mathrm{~mm}$ in women and $41-46 \mathrm{~mm}$ in men, moderate enlargement for $43-46 \mathrm{~mm}$ in women and $47-51 \mathrm{~mm}$ in $\mathrm{men}$, and severe enlargement for $\geq 47 \mathrm{~mm}$ in women and $\geq 52 \mathrm{~mm}$ in men. Left ventricular ejection fraction (LVEF) was calculated using the biplane method of discs (modified Simpson's rule) [5].

All data are presented as mean \pm standard deviation (SD) for parametric variables and as percentages for categorical variables. Continuous variables were checked for the normal distribution assumption using the Kolmogorov-Smirnov statistics. Differences between the prespecified patient groups were evaluated using the Kolmogorov-Smirnov test or ANOVA with Tukey's post hoc test as appropriate. Categorical variables were tested by Pearson's $\chi^{2}$ test and Fisher's exact test. A p-value $<0.05$ was considered statistically significant. All statistical studies were carried out using Statistical Package for Social Sciences software (SPSS 21.0 for macOS, SPSS Inc., Chicago, IL, USA).

\section{Results}

The study population consisted of 113 individuals with $\mathrm{AF}$ (mean age $77.2 \pm 9.8$ years; $37.2 \%$ men). In this group, the mean value of LA diameter was 42.2 \pm 8.2 millimeters. Hypertension was present in 97 (85.4\%) patients, diabetes mellitus in $39(34.5 \%)$ patients, chronic coronary syndromes in 43 (38\%) patients, stroke or transient ischemic attack in 22 (19.5\%) patients, HF in 51 (45.1\%) patients and chronic obstructive pulmonary disease in 15 (13.3\%) patients. Baseline characteristics of study patients are shown in Table I.

In the group of $\mathrm{AF}$ patients, we identified 71 $(62.8 \%)$ patients with LAE (age $77.6 \pm 9.9$ years; $36.6 \%$ men) and $42(37.2 \%)$ patients without LAE (76.6 \pm 9.7 years; $33.3 \%$ men). There was no significant difference in the incidence of hypertension, diabetes mellitus, chronic coronary syndromes, chronic kidney disease, stroke or transient ischemic attack, as well as chronic obstructive pulmonary disease in these two groups. The incidence of HF was significantly higher in AF patients with LAE [39 (54.9\%) compared to the patients without LAE $-12(28.6 \%), p=0.01]$. The characteristics of the study population according to the LAE status are shown in Table II. 
Table I. Baseline characteristics of patients with atrial fibrillations (AF)

\begin{tabular}{|l|c|}
\hline & $\begin{array}{c}\text { Overall patient } \\
\text { population } \\
\text { (n = 113) }\end{array}$ \\
\hline Age (yrs) & $77.2 \pm 9.8$ \\
\hline Male [n (\%)] & $42(37.2)$ \\
\hline BMI [kg/m²] & $29.1 \pm 4.9$ \\
\hline Obesity [n (\%)] & $33(29.2)$ \\
\hline SBP [mm Hg] & $133.4 \pm 20.6$ \\
\hline DBP [mm Hg] & $80 \pm 13.6$ \\
\hline IVSd [mm] & $13.1 \pm 2.6$ \\
\hline LAE [n (\%)] & $71(62.8)$ \\
\hline Lad [mm] & $42.2 \pm 8.2$ \\
\hline Hypertension [n (\%)] & $97(85.4 \%)$ \\
\hline Diabetes mellitus [n (\%)] & $39(34.5 \%)$ \\
\hline Chronic coronary syndromes [n (\%)] & $43(38 \%)$ \\
\hline Stroke, TIA [n (\%)] & $22(19.5 \%)$ \\
\hline Heart failure [n (\%)] & $51(45.1 \%)$ \\
\hline COPD [n (\%)] & $15(13.3 \%)$ \\
\hline $\begin{array}{l}\text { BMl - body mass index; IVSd - intraventricular septum diameter; SBP - systolic blood pressure; } \\
\text { DBP- diastolic blood pressure; LAd - left atrium diameter; TIA- transient ischemic attack; } \\
\text { COPD - chronic obstructive pulmonary disease }\end{array}$ \\
\hline
\end{tabular}

\section{Discussion}

The assessment of LA size is crucial to determine the LA function. During the cardiac cycle, LA size is changing and in clinical practice, the maximum LA size (at the end of the left ventricle systole) is the most often used parameter. The measurement of anteroposterior LA linear dimension by $2 \mathrm{D}$ echocardiography in the parasternal long-axis view remains the most common echocardiographic method, because of its simplicity and reproducible.

Left atrial enlargement reflects structural remodeling associated with pathophysiologic processes in different cardiovascular diseases (CVD). Conditions such as hypertension, HF, diabetes mellitus, and obesity are the most common risk factors of LA remodeling. In hypertensive patients, associated hypertrophy and diastolic dysfunction lead to LEA. Left atrial enlargement is not the only consequence of high blood pressure, but also a predictor of outcome in hypertension [6]. Hypertension increases the risk of AF [7]. In our population of AF patients, hypertension was observed in most patients $(85.4 \%)$, without significant differences between patients with and without LAE.

Previous studies have shown that LA size has been associated with adverse cardiovascular outcomes. Left atrial enlargement has been described
Table II. Baseline characteristics of the study population according to the status of left atrial enlargement (LAE)

\begin{tabular}{|c|c|c|c|}
\hline & $\begin{array}{c}\text { LAE }+ \\
(n=71)\end{array}$ & $\begin{array}{l}\text { LAE- } \\
(n=42)\end{array}$ & $\mathrm{p}$-value \\
\hline Age (yrs) & $77.6 \pm 9.9$ & $76.6 \pm 9.7$ & $>0.05$ \\
\hline Male [n (\%)] & $26(36.6 \%)$ & $14(33.3)$ & $>0.05$ \\
\hline $\mathrm{BMI}\left[\mathrm{kg} / \mathrm{m}^{2}\right]$ & $29.7 \pm 5.3$ & $28.1 \pm 4.1$ & $>0.05$ \\
\hline Obesity [n (\%)] & $24(33.8)$ & $9(21.4)$ & $>0.05$ \\
\hline $\mathrm{SBP}[\mathrm{mm} \mathrm{Hg}]$ & $134 \pm 21.8$ & $132.4 \pm 18.7$ & $>0.05$ \\
\hline $\mathrm{DBP}[\mathrm{mm} \mathrm{Hg}]$ & $81.4 \pm 14.1$ & $77.1 \pm 12.7$ & $>0.05$ \\
\hline IVSd [mm] & $13.1 \pm 2.6$ & $12.4 \pm 2$ & $>0.05$ \\
\hline $\operatorname{LVEF}(\%)$ & $53.5 \pm 13.8$ & $57.1 \pm 10.1$ & $>0.05$ \\
\hline Hypertension [n (\%)] & $60(84.5)$ & $37(88.1)$ & $>0.05$ \\
\hline $\begin{array}{l}\text { Diabetes mellitus } \\
{[\mathrm{n}(\%)]}\end{array}$ & $26(36.6)$ & $13(31)$ & $>0.05$ \\
\hline $\begin{array}{l}\text { Chronic kidney } \\
\text { disease [n (\%)] }\end{array}$ & $25(35.2)$ & $11(26.2)$ & $>0.05$ \\
\hline $\begin{array}{l}\text { Chronic coronary } \\
\text { syndromes [n (\%)] }\end{array}$ & $28(39.4)$ & $15(35.7)$ & $>0.05$ \\
\hline Stroke, TIA [n (\%)] & $11(15.5)$ & $11(26.2)$ & $>0.05$ \\
\hline Heart failure [n (\%)] & $39(54.9)$ & $12(28.6)$ & $=0.01$ \\
\hline COPD [n (\%)] & $10(14.1)$ & $5(11.9)$ & $>0.05$ \\
\hline
\end{tabular}

AF — atrial fibrillation; BMI — body mass index; SBP — systolic blood pressure; DBP — diastolic blood pressure; LAE - left atrial enlargement; IVSd - intraventricular septum diameter; LVEF — left ventricular ejection fraction; TIA — transient ischemic attack; COPD — chronic obstructive pulmonary disease

as a predictor of $\mathrm{AF}$, stroke, congestive $\mathrm{HF}$, and cardiovascular death. In our study, LAE was highly prevalent in nonvalvular AF patients (almost 62.8\% of patients). Echocardiographically determined LA size is one of the stroke risk factors overlooked by the scores used for thromboembolic risk stratification. LA size may be an additional predictor useful in the assessment of thromboembolic events in AF patients. As we presented in our previous study, LAE has been associated with higher thromboembolic risk assessed by CHADS2 and CHA2DS2VASc scores [4].

The clinical implications of LAE can be left ventricular dysfunction, which occurs in $20-30 \%$ AF patients [2]. Left atrial enlargement has been shown as a marker of poor prognosis in HF patients. Previous studies have demonstrated that LA size is a powerful predictor of outcome among HF patients with predominantly impaired systolic function and provides additional prognostic information beyond left ventricular systolic and diastolic function. (3) The results of our study showed, that the prevalence of $\mathrm{HF}$ was significantly higher in AF patients with LAE compared to AF patients without LAE. What is important, the mean value of LVEF did not differ significantly between these two groups. 
Limitations in this study were a small population of the study, one center study and the lack of comparison of different methods used for assessing LA size.

\section{Conclusions}

Left atrial enlargement is highly present in the Polish population of AF patients and can carry important clinical and prognostic implications. Patients with $\mathrm{AF}$ and LAE are at higher risk of HF. Left atrial size can also have prognostic value. Further studies sufficiently powered to assess this issue may be needed.

\section{Conflict of interest}

The authors have no conflicts of interest to disclose.

\section{References}

1. Hoit BD. Left atrial size and function: role in prognosis. J Am Coll Cardiol. 2014; 63(6): 493-505, doi: 10.1016/j.jacc.2013.10.055, indexed in Pubmed: 24291276.

2. Kirchhof P, Benussi S, Kotecha D, et al. ESC Scientific Document Group. 2016 ESC Guidelines for the management of atrial fibril- lation developed in collaboration with EACTS. Eur Heart J. 2016; 37(38): 2893-2962, doi: 10.1093/eurheartj/ehw210, indexed in Pubmed: 27567408 .

3. Rossi A, Temporelli PL, Quintana M, et al. MeRGE Heart Failure Collaborators. Independent relationship of left atrial size and mortality in patients with heart failure: an individual patient meta-analysis of longitudinal data (MeRGE Heart Failure). Eur J Heart Fail. 2009; 11(10): 929-936, doi: 10.1093/eurjhf/hfp112, indexed in Pubmed: 19789395 .

4. Hrynkiewicz-Szymanska A, Dluzniewski M, Platek AE, et al. Association of the CHADS2 and CHA 2DS 2-VASc scores with left atrial enlargement: a prospective cohort study of unselected atrial fibrillation patients. J Thromb Thrombolysis. 2015; 40(2): 240-247, doi: $10.1007 / s 11239-014-1154-6$, indexed in Pubmed: 25490871

5. Lang RM, Bierig M, Devereux RB, et al. American Society of Echocardiography's Nomenclature and Standards Committee, Task Force on Chamber Quantification, American College of Cardiology Echocardiography Committee, American Heart Association, European Association of Echocardiography, European Society of Cardiology. Recommendations for chamber quantification. Eur J Echocardiogr. 2006; 7(2): 79-108, doi: 10.1016/j.euje.2005.12.014, indexed in Pubmed: 16458610 .

6. Mancusi C, Canciello G, Izzo R, et al. Left atrial dilatation: A target organ damage in young to middle-age hypertensive patients. The Campania Salute Network. Int J Cardiol. 2018; 265: 229-233, doi: 10.1016/j.ijcard.2018.03.120, indexed in Pubmed: 29628278.

7. Tsioufis C, Konstantinidis D, Nikolakopoulos I, et al. Biomarkers of Atrial Fibrillation in Hypertension. Curr Med Chem. 2019; 26(5): 888-897, doi: 10.2174/0929867324666171006155516, indexed in Pubmed: 28990508. 